\title{
Health Information Availability and Utilization by Medical Practitioners for Chronic Disease Management in Central Hospitals, Warri-Delta State
}

\author{
${ }^{1,}$ Rev. Dr Obiora Nwosu (PhD)-CLN, ${ }^{2}$ Dr. Esoswo Francisca Ogbomo (PhD), \\ ${ }^{3}$ Dr E.S Anaehobi $(\mathrm{PhD})$

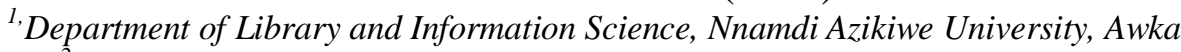 \\ ${ }^{2}$, Department of Library and Information Science,Delta State University, Abraka \\ ${ }^{3,}$ Department of Library and Information Science,Nnamdi Azikiwe University, Awka
}

\begin{abstract}
In developing countries like Nigeria, large proportions of the population have limited access to health information and health care facilities. Thus there is the fear that health professionals and patients may not be aware of the availability of health information in developing countries like Nigeria, large proportions of the population have limited access to health information and health care facilities. Thus there is the fear that health professionals and patients may not be aware of the availability of health information as well as their utilization with regards to the management of chronic diseases. The research design that was utilized for this study is the survey design type of descriptive research. The research instrument that was used to collect data for the study is questionnaire. The following findings were derived from this study: Majority of the health professionals affirmed that there is health information policy that they are expected to adhere to and also they agreed that they offered health information and education to all categories of patients, on the nature of health information. This notwithstanding, there are general lack of awareness on the need for health information to the public for the prevention and management of chronic diseases in Delta State, Nigeria.
\end{abstract}

Keywords: Chronic disease management; Medical Practitioners; Health Information availability and utilization; Hospitals.

\section{INTRODUCTION}

In developing countries like Nigeria, large proportions of the population have limited access to health information and health care facilities. Equitable access to health care remains a challenge in developing and transitional countries, especially among the rural poor. Pharmacies in densely populated areas are always more lucrative, often leaving sparsely-populated rural regions without access to reliable sources of health care. There are chronic diseases which are terminal illness. They require comprehensive health information for their management. Thus there is the fear that health professionals and patients may not be aware of the availability of health information as well as their utilization with regards to the management of chronic diseases. Consequently, the study is set out to investigate the availability and utilization of health information by health professionals for chronic disease management in Central Hospital Warri, Delta State.

\section{Research Questions}

[1] To what extent is Health Information for Chronic Diseases Management Available to Health Professionals?

[2] What are the sources of Health Information for Chronic Diseases Management?

[3] Do the hospital/health professionals provide chronic diseases self management education to patients?

\section{LITERATURE REVIEW}

Grilli, Ramsay and Minozzi (2002 as cited by WHO, 2011) defined chronic diseases as "diseases of long duration and generally slow progression". The United State Centres for Disease Prevention and Control defined chronic diseases as "condition that are not cured once acquired". Chronic disease simply means persistent or recurring disease, usually affecting a person for three months or longer (Swider, 2002). A chronic disease is generally one that is hereditary or one that is the result of factors such as poor diet and living Conditions, using tobacco or other harmful substances, or a sedentary lifestyle. Such a disease is not typically contracted from another person by contagion, because most chronic illness are not caused by infection. The term chronic disease commonly applies to conditions that can be treated but not necessarily cured. There are different types of chronic disease from A to Z. From Aizheimer's, arthritis and asthma to "zoo noses", According to Morrison (2002), the most common chronic diseases in the European Region are heart disease, stroke, cancer, 
respiratory disease, diabetes and mental health problems. Health Grade Inc. (2011) listed the following as types of chronic diseases. They include:

- Heart disease.

- Diabetes

- Cardiovascular Disease

- Cerebrovascular Disease

- Hypertension

- Kidney Disease

- Liver Disease

- Alzheimer's Disease

- Arthritis

- Cancer

- Chronic Obstructive Lung Disease

- Epilepsy

- Hereditary Hemochromatosis

- Iron Overload

- Oral Diseases

- Osteoporosis

\subsection{Sources of health information for patients with chronic diseases}

The costs and effects of chronic disease are significant, but chronic disease can be reduced or onset prevented until much later in life. Making changes does not have to be expensive, and the means of preventing and controlling most chronic diseases are already well know. These range from interventions that target individuals or families (such as providing information leaflets and proactive telephone support), initiatives aimed at health professionals (such as decision support tools, training for new roles and multidisciplinary teams), organizational change (such as workplace education and intersectoral work) and strategies aimed at populations (such as health promotion advertising and identifying people at different levels of risk (Gohler et al., 2006). Gohler et al further noted that rather than focusing on certain interventions alone, chronic disease management is a way of coordinating care and ensuring that people gain the support they need at an appropriate time. Evidence suggests that planned, proactive care can lead to identifying people at different levels of risk. Rather than focusing on certain intervention alone, chronic disease management is a way of coordinating care and ensuring that people gain the support they need at an appropriate time; evidence suggests that planned, proactive care can lead to a better quality of life and improved health outcomes for people with chronic diseases.

\subsection{Provision of chronic diseases self-management education by hospitals/health professionals}

Chronic Care Management (CCM) is a proven approach for people with specific progressive conditions, ones where progression can be significantly reduced by straightforward and usually inexpensive interventions. Modifiable conditions include diabetes, unstable angina, ischemic heart disease, chronic congestive heart disease and COPD or "smoker's lung", and asthma (Jeffs, 2004).CCM approaches acknowledge that current methods of healthcare delivery are more equipped to cater for acute conditions than the more holistic management of chronic conditions. Conditions such as diabetes and ischemic heart disease need to be managed proactively throughout the disease spectrum, from measures to prevent the onset of the condition, through to solving progression and avoiding complications. Ways of achieving the encompass regular reviews, use of evidence based best practice care alternative models of service delivery that often involve more care in the community. One significant enabler for chronic care management will be the intelligent application of information technology within healthcare. Examples of this include electronic clinical decision support tools, electronic prompts, reminders, electronic patient records, and data analysis capability to support planning and population health initiatives.

According to Jose, Santiago, Eugenio, and Alvar (2005) chronic conditions, unlike acute diseases, cannot be cured and are likely to last more than a year, limit what one can do, and require ongoing medical attention. In the U.S., chronic conditions affect about 125 million people and are the leading cause of illness, disability, and death. An estimated 60 million people have two or more chronic conditions. Jose, Santiago, Eugenio, and Alvar further noted that new models of chronic disease care have potential to reduce urgent and emergency care and reduce the side effects of chronic disease and non-acute conditions, thus leading to an improved quality of life and significant cost savings in the total care of patients. Diabetes is perhaps the bestknown success story. People with diabetes need to be educated in the management of their condition, to monitor their blood sugar levels, and to use diet, exercise, medications, and injections of insulin to minimize the impact 
of diabetes on their health. Sleep apnea, a common sleep disorder and chronic obstructive pulmonary disease (COPD) are also chronic/non-acute conditions that respond to continuing daily care and management by the patient. People with chronic conditions can get better health outcomes when they participate in managing their health care. Health care for non-acute/chronic conditions cannot be effective when it takes place only in a "medical" setting. It must take place wherever the patient is and at all times. Therefore, instead of health care taking place solely in the doctor's office, it is essential to change the location, timing, and frequency of interaction between medical professionals and their patients. In addition to a medical team which receives patients in the office, seeing any one patient only a few times each year, a patient can also have frequent contact with a medical professional without going into the office. The patient needs information, support, and timely communication with health care professionals, while the medical team needs communication with the patient and his/her support network in order to provide timely advice or intervention. Innovative programs will involve disease management, telemedicine, Internet community, and consumer medical informatics. The changes in interaction mediated by these technologies and methods may be a bridge across the office/community boundary with the potential for improving the nature of relationships in health care (Kapur, Strohl, Redline, Iber, O'Connor \& Nieto, 2002).

Kapur et al further indicated that the challenge is to support and lead the emerging standard of chronic disease management. New systems are needed to integrate information into systems for training and education; to enable and support culture change; and to enhance new patterns of interaction and information use. The health care system needs complementary services and capabilities to enable enhance relationships among health professionals, patients, families, and other professional and lay personnel. New models of care, based on medically proven principles, can improve the health and well-being of patients, and needs to encompass training, education, publications, and consulting for physicians, health professionals, patients, and their families. Caregivers need to learn how to organize a full regimen of care that may span relatives, friends, and community services as well as hospitals, home health care, and social service agencies. Coordination and communication of health information are vital.

\section{METHODOLOGY}

Descriptive survey was employed for the study. Thirteen (13) respondents were randomly selected for the study. This consists of all professional health workers in the central hospital, Warri. Questionnaire was the instrument used for data collection. The data were analyzed using descriptive statistics i.e. Percentages and frequencies.

\subsection{Findings and Discussion}

A total of 13 copies of the questionnaire were distributed and 10 i.e. $77 \%$ were returned. The response rate of (77\%) is considered adequate for the study because the standard and acceptable response rate for most studies is 60\% (Malaney, 2002: Evans, Peterson, \& Demark-Wahnefried, 2004, as cited in Dulle, MinishMajanja \& Cloete, 2010).

\section{Bio-Data of the Respondents}

Table I: Sex Distribution of Health Professionals

\begin{tabular}{|lcc|}
\hline Sex & Frequency & Percentage $(\boldsymbol{\%})$ \\
\hline Male & 3 & 30 \\
Female & 7 & 70 \\
\hline Total & 10 & 100 \\
\hline
\end{tabular}

As can be seen from Table I above, there are more female Health professionals than male in the Central Hospital Warri, Delta State.

Table II: Distribution of Health Professionals by profession

\begin{tabular}{|lcc|}
\hline Age & Frequency & Percentage (\%) \\
\hline Medical health professionals & 2 & 20 \\
Nurse & 8 & 80 \\
Pharmacist & - & - \\
Physiotherapist & - & - \\
\hline Total & 10 & 100 \\
\hline
\end{tabular}

Table II shows that there are more nurses $8(80 \%)$ and $2(20 \%)$ medical health professionals. The result shows that there are no pharmacist and physiotherapist. 


\section{Answering of the Research Questions}

Research Question I: To what extent is Health Information for Chronic Diseases Management Available to Health Professionals?

All the health professionals affirmed that there is Health Information policy that they are expected to adhere to. They also agreed that they offered health Information and education to patients, and that their target for health information is to all categories of patients. The health professionals were also asked to indicate the nature of health information they give to patients. Their mean response is presented in Table III below.

Table III: Mean Response of the Nature of Health Information Health Professionals give to Patients

\begin{tabular}{|l|c|c|}
\hline \multirow{2}{*}{} & N & Mean \\
\cline { 2 - 3 } & Statistic & Statistic \\
\hline NATURE OF & 10 & 4.12 \\
HEALTH & 10 & \\
INFORMATION & & \\
Valid N (listwise) & & \\
\hline
\end{tabular}

Criterion mean $=\mathbf{3 . 0 0}$

With a mean statistic of 4.12 which is well above the criterion mean of 3.00, it shows that health professionals give health information to patients. Some of the health given are name and nature of the illness, how to cope with the illness, name of prescribed drugs and possible side effects of the drugs.

Research Question 2: What are the sources of Health Information for Chronic Diseases Management?

Table IV: Sources of Health Information for Chronic Diseases Management by health professionals

\begin{tabular}{|lcc|}
\hline Sources & Frequency & Percentage (\%) \\
\hline Internet & 2 & 20 \\
Library & 3 & - \\
Other relevant hospital dept. & - & - \\
Newspaper/TV & - & 20 \\
Patients support groups & 2 & 30 \\
Invisible college system & 3 & $\mathbf{1 0 0}$ \\
\hline Total & $\mathbf{1 0}$ & \\
\hline
\end{tabular}

From the Table IV above it shows that health professionals access health information from relevant sources and resources for the management of chronic diseases. They make use of the library internet, patients support groups and the invisible college system (conferences, seminars, etc.).Research Question 3: Do the hospital/health professionals provide chronic diseases self management education to patients?

Table V: Self Management Education to Patients with Chronic Diseases.

\begin{tabular}{|c|c|c|}
\hline Self management & Frequency & Percentage (\%) \\
\hline Group training & 6 & 25.0 \\
\hline Individualized counseling & 8 & 33.3 \\
\hline Information guidebooks/pamphlets & 5 & 20.8 \\
\hline Multi-media demonstration & 2 & 8.3 \\
\hline Attendance to self help group meetings & 1 & 4.2 \\
\hline
\end{tabular}

In Table $\mathrm{V}$ above, it can be deduced that group training, individualized counseling and information guidebooks/pamphlets are some of the self management education programs provided by health professionals to patients with chronic diseases. The results derived from the analysis shows that there are more female than male health professionals and majority of the health professionals are Nurses. However, there are few medical professionals. Majority of the health professionals affirms that there is health information policy that they are expected to adhere to and also they agreed that they offered health information and education to patients. 
This supports the work of Kapur, Strohl, Redline, Iber, O'Connor \& Nieto, 2002) who indicated that the challenge is to support and lead the emerging standard of chronic disease management. New systems are needed to integrate information into systems for training and education; to enable and support culture change; and to enhance new patterns of interaction and information use. The health care system needs complementary services and capabilities to enable enhanced relationships among health professionals, patients, families, and other professional and lay personnel. New models of care, based on medically proven principles, can improve the health and well-being of patients, and needs to encompass training, education, publications, and consulting of physicians, health professionals, patients, and their families. Caregivers need to learn how to organize a full regimen of care that may span relatives, friends, and community services as well as hospitals, home health care, and social services agencies.

On the nature of health information, the health professionals gives information on names/nature of the illness, how to cope with the illness, name of prescribed drugs and possible side effects of the drugs. Also on method of provision of health information, it shows that poster and handbills, organization of relevant department seminars, etc. community health information outreach programmes are the methods of provision of health information. This finding supports the work of Jose, Santiago, Eugenio, and Alvar (2005) who noted that new models of chronic care have the potential to reduce urgent and emergency care and to reduce the side effects of chronic disease and non-acute conditions, thus leading to an improved quality of life and significant cost savings in the total care of patients. Diabetes is perhaps the best-known success story. People with diabetes need to be educated in the management of their condition, to monitor their blood sugar levels, and to use diet, exercise, medications, and injections of insulin to minimize the impact of diabetes on their health. Sleep apnea, a common sleep disorder and chronic obstruction pulmonary disease (COPD) are also chronic/non-acute conditions that respond to continuing daily care and management by the patient.

Group training, individualized counseling and information guidebooks/pamphlets are some of the self management education programmes provided by health professionals to patients with chronic diseases. This is in line with Gohler, et al. (2006) who noted that the costs and effects of chronic disease are significant, but chronic disease can be reduced or onset prevented until much later in life. Making changes does not have to be expensive, and the means of preventing and controlling most chronic diseases are already well known. These range from interventions that target individuals or families (such as providing information leaflets and proactive telephone support), initiatives aimed at health professionals (such as decision support tools, training for new roles and multidisciplinary teams), organizational change (such as workplace education and intersectoral work) and strategies aimed at populations such as health promotion advertising and identifying people at different levels of risk (Gohler et al., 2002). Gohler et al. noted that rather than focusing on certain interventions alone, chronic disease management is a way of coordinating care and ensuring that people gain the support they need at an appropriate time.

\section{Conclusion and Recommendations}

The study concludes that health information on chronic diseases should be made available to patients with chronic diseases to enable them understand and manage themselves. This will in turn prolong their life span.

Based on the findings of this study, the following recommendations were made:

[1] There is the need for the health professionals to adhere to the information policies provided by the hospital to enable health professional provide adequate self management of chronic diseases.

[2] Health professionals and other health professionals should educate the patients on the name and nature of their illness, how to cope with the illness, name of prescribed drugs and possible side effects of the drugs.

[3] Sensitization programmes should be organized by the hospital to bring about awareness of the services rendered in the central hospital, Warri.

\section{REFERENCES}

Dulle, F.W. \& Minish-Majanja, M.K. \& Cloete, L.M. (2010). Factors influencing the adoption of open access scholarly communication in Tanzania public universities. World Library and Information Congress: $76^{\text {th }}$ IFLA General Conference and Assembly 10-15 August 2010, Gothenburg, Sweden. Retrieved September 1, 2010 from http:// www.ifla.org/en/ifla76

[2] Gohler, A. et al. (2006). A systematic meta-analysis of the efficacy and heterogeneity of disease management programmes in congestive heart failure. Journal of Cardiac Failure, 12, 554-567

[3] Grilli, R, Ramsay, C, Minozzi, S. (2002). Mass media interventions: effects on health services utilization. Cochrane Database of Systematic Reviews (I): DOI: 10.1002/14651858.CD000389. Retrieved October 30, 2011, from http://www2.cochrane.org/reviews/en/ab000389.html

[4] Health Grades Inc. (2011). Chronic illness: Types list. Retrieved October 30, 2011,

from http://www.rightdiagnosis.com/c/ chronic/subtypes.htm 
[5] Jeffs, T (2004). Midland Regional Diabetes Strategy. Midland District Health. Retrieved October 30, 2011, From www.tdhb.org.nz/.../chronic disease management requirements.pdf

[6] Jose, M. M., Santiago, J.C., Eugenio, V. and Alvar, G. N.A. (2005). Long-term cardiovascular outcomes in men

with obstructive sleep apnoea-hypopnoea with or without treatment with continuous positive airway pressure: an observational study. Lancet; 365, 1046-1053.

[7] Kapur, V., Strohl, K.P., Redline, S., Iber, C., O’Connor, G. and Nieto, J. (2002). "Under Diagnosis of sleep apnea syndrome in U.S. Communities, "Sleep Breath, 6 (2), 49-54.

[8] Morrison, A. (2001). Effectiveness of printed patient educational materials in chronic illness: a systematic Review of controlled trials. Journal of Managed Pharmaceutical Care, 1, 51-62.

[9] Swider, S.M. (2001). Outcome effectiveness of community health workers: an integrative literature review. Public Health Nursing, 19, 11-20.

[10] WHO (2011). Working paper on data sources, methods, results for projections of morality and burden of disease for 2005, 2015, 2030. Retrieved October 29, 2011, from http://www.who.int/chp/chronic_disease_report/en/ 